\title{
DESIGN AN AR APPLiCATION IN FINDING Preferred Dining Place With Social NETWORK CAPABILITY (ARAFEPS)
}

\author{
Farhat $^{1}$, Avinanta Tarigan ${ }^{2}$, Remi Senjaya ${ }^{3}$ \\ ${ }^{1}$ Department of Informatics Engineering, Faculty of Technology Industry \\ Gunadarma University, West Java, Indonesia \\ farhat_nus.salnaz@student.gunadarma.ac.id \\ ${ }^{2}$ Department of Informatics Engineering, Faculty of Technology Industry \\ Gunadarma University, West Java, Indonesia \\ avinanta@staff.gunadarma.ac.id \\ ${ }^{3}$ Department of Informatics Engineering, Faculty of Technology Industry \\ Gunadarma University, West Java, Indonesia \\ remilstaff.gunadarma.ac.id
}

\begin{abstract}
Location Based Service (LBS) application help people in finding places or point of interest. However, spatial information in LBS is presented as a map in a small mobile device screen which makes the user difficult to understand and to find the location they need. It is possible that user might have disorientation in finding the place although they are presented with correct map. ARAFEPs (Augmented Reality Assistant to Finding Preferred Dining Place) is an Augmented Reality application to support its user to find the nearest and preferred dining place such as restaurant, fast food and food court. It is developed using Layar $A R$ as platform. It presents the user with spatial information in a way that user could aware and understand the places surround them. Additional and relevant information that are presented in AR aids users to decide which dining place fits the needs of the user.
\end{abstract}

\section{KEYWORDS}

Layar, Layer, Augmented Reality, POI

\section{INTRODUCTION}

Everyone definitely need dining places to fulfill their basic needs whether it in the Restaurant and Non Restaurant. But for someone who was in an unfamiliar place or in a crowd, then to know and find an dining place that are in the surrounding is not easy. With the rapid growing of the technology, this problem can be resolved. We can easily find the dining places that are around us only with the help of smartphone that we always carry.

With seeing the problems that exist and problem that it is difficult for ordinary user to develop spatial awareness if only based on the LBS concepts that presented in a map view in a small mobile device screen, The author developed an application that can find dining places that was around user by AR visualization. So users can view the information in a more tangible. With this application, the user can specify an dining place that matching with his needs. In addition, with this application users can be take active role. Users can post their existence in a particular dining place into the database so it can facilitate access to the data useful additions also as a reference for 
other users. It can be done directly on the users smartphone camera screen. instructions may often appear daunting, the simplest approach is to use this template and insert headings and text into it as appropriate.

\section{LIRETATURE REVIEW}

\subsection{LBS (Location Based Service)}

LBS application use the geolocation functionality of a mobile phone or smart phone to provide people with information and entertainment. LBS are used in a variety of contexts, such as health, indoor object search, entertainment, work, personal life, etc [1]. Basic questions that LBS users are concerned about include: Where am i currently? What and where are the nearest locations of interest? How to get there? [2].

\subsection{AR (AUgMENTED REALITY)}

The Augmented Reality (AR) can also be said as a variation of Virtual Environments (VE). VE technologies completely immerse a user inside a synthetic environment. While immersed, the user cannot see the real world around him. In contrast, AR allows the user to see the real world, with virtual objects superimposed upon or composited with the real world. Therefore, AR supplements reality, rather than completely replacing it. AR defined as systems that have three characteristics such as Combines real and virtual, Interactive in real time, Registered in 3-D [3].

AR itself aims to complete information on a real object that can not be captured by the human senses either through the sense of hearing, touch so helps user carry out activities in the real world. The most popular type of AR application for mobile devices available today is the browser that overlays contextual data about objects or locations for user surroundings. The real strength of AR browsers is their discoverability. Today, browsers have most of the attention and it's amazing how many people have yet to experience a browser for themselves. Browsers are incredibly useful ways to discover information about places and objects around us. Browsers have helped many user discover information about user neighborhood that their never would have discovered otherwise [4].

\subsection{LAYAR}

Layar specializes in mobile augmented reality because it is most popular medium through which the average person interacts with AR content. Layar is an open platform for third-party content [5]. Layar provides two types of AR services which specialize in Geolocation-based and Visionbased. Geolocation-based AR uses GPS, compass and other sensors in a user's mobile phone to provide a "heads-up" display of various geolocated points-of-interest. Vision-based AR uses many of these same sensors to virtually display digital content in context with real-world objects like magazines, postcards or product packaging - by tracking the visual features of these objects.

\subsection{Usecase Diagram}

Usecase diagram is a list of steps, typically defining interactions between a role (known in UML as an "actor") and a system, to achieve a goal. The actor can be a human or an external system. In a use case diagram are terms such as actors, use cases and use case relationships. 


\subsection{Class Diagram}

Class diagram is a type of static structure diagram that describes the structure of a system by showing the system's classes, their attributes, operations (or methods) and the relationships among the classes. In the diagram, classes are represented with boxes which contain three parts [6] :

- The upper part holds the name of the class.

- The middle part contains the attributes of the class.

- The bottom part gives the methods or operations the class can take or undertake.

\subsection{Flowchart}

A flowchart is a type of diagram that represents an algorithm or process, showing the steps as boxes of various kinds, and their order by connecting these with arrows. Process operations are represented in these boxes, and arrows connecting them represent flow of control. Flowcharts are used in designing and documenting complex processes or programs. Like other types of diagram, they help visualize what is going on and thereby help the viewer to understand a process, and perhaps also find flaws, bottlenecks, and other less-obvious features within it [7].

\section{Analysis and Design}

\subsection{User Requirement Analysis}

Functional Requirements :

- The application should displays information about dining places around the user that consists of the dining place's name, address, provided food, type's icon and distance between the user and each dining places. Based on the maximum distance that has been selected and based on user coordinates position (lat, lon).

- This application provide the action to add new POI. To ensure the validity of the data that are submitted, the application classifies the user into member and non member. The actions that provided by the system for member are : calling to the dining place, send email, accessing the official website, twitter and facebook of the dining place, add new POI, and logout as non member. For non member, the actions are same with the actions for member except the actions to add new POI data and logout.

- The applications provide action for member to add new POI data about the dining place being visited such as name of the dining place, the foods that are sold, address, telephone number, official website, facebook, twitter page, email, type of the dining place and the position of the user (by the system).

\subsection{Design}

\subsubsection{The Architecture of Layar}

1. The Layar Reality Browser : The mobile device of the user.

2. The Layar Server : Provides the interfaces to the Layar Reality Browser, the Layar Publishing Website and the external Layar Service Providers.

3. The Layar Publishing Website : The website where the Layer admin can apply the settings to their layer. 
4. The Layer Service Providers : Webservice by layer (ARAFEPs) developer which contains all data / information required by the layer to run in accordance with its goal.

\subsubsection{Application Design}

In this section through a visual depiction of how the interaction between the user which consist of member and non member with the system, admin with the back end system and how the system is running are explained. Visually design is explained using with using software DIA version 0.97.2.

\subsubsection{Usecase Diagram}

Use case diagrams explaining how users interact with the system from the application made. There are three models of interaction are distinguished, that are:

\subsection{Usecase diagram for the Admin}

Figure 1 is intended for the admin. Seen that the Admin can do :

- Login into his webserver account.

- Insert, delete, update (edit) dining places data to or from the Data_POI table.

- Create action table by entering the name of the action, action information, content type (type of action) and determine / linking the POI data which accordance with the existing actions.

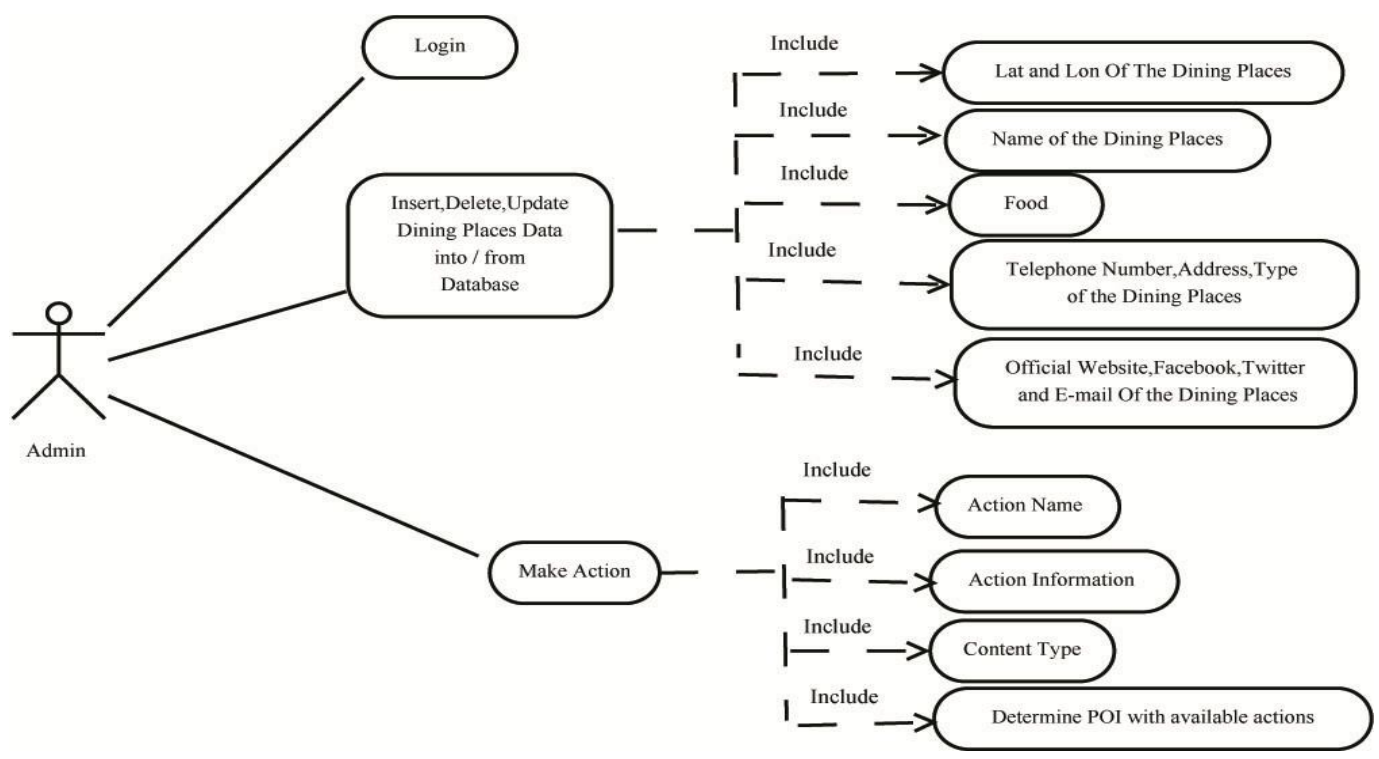

Figure 1: Usecase diagram for the Admin

\subsection{Usecase Diagram for Member}

This application has an user categorizations in using the service / menu provided. For a member / user that have been registered and have ID then the offered services facility more than the user that does not include member / do not have an ID / not registered. From the Figure 2 can be seen that a member can do : 
Advanced Computing: An International Journal (ACIJ ), Vol.4, No.4, July 2013

- Login.

- Seeing the nearest dining places where its parameters are the coordinates of the user position, the maximum distance specified by user and POI Request from the system.

- Adding new data that consists of :

$\circ$ Name of dining place.

$\circ$ Address of dining place.

- Food provided.

- Telephone number of the dining place.

- Official website of the dining place.

- Twitter and Facebook account of the dining place.

$\circ$ Dining place's E-Mail.

- As well as the type of dining place (Restaurant / Non restaurant).

- User position coordinates from GPS.

- Help to open the Help page.

- Layer Info to open pages that describes the application.

- LogOut to exit from the member status.

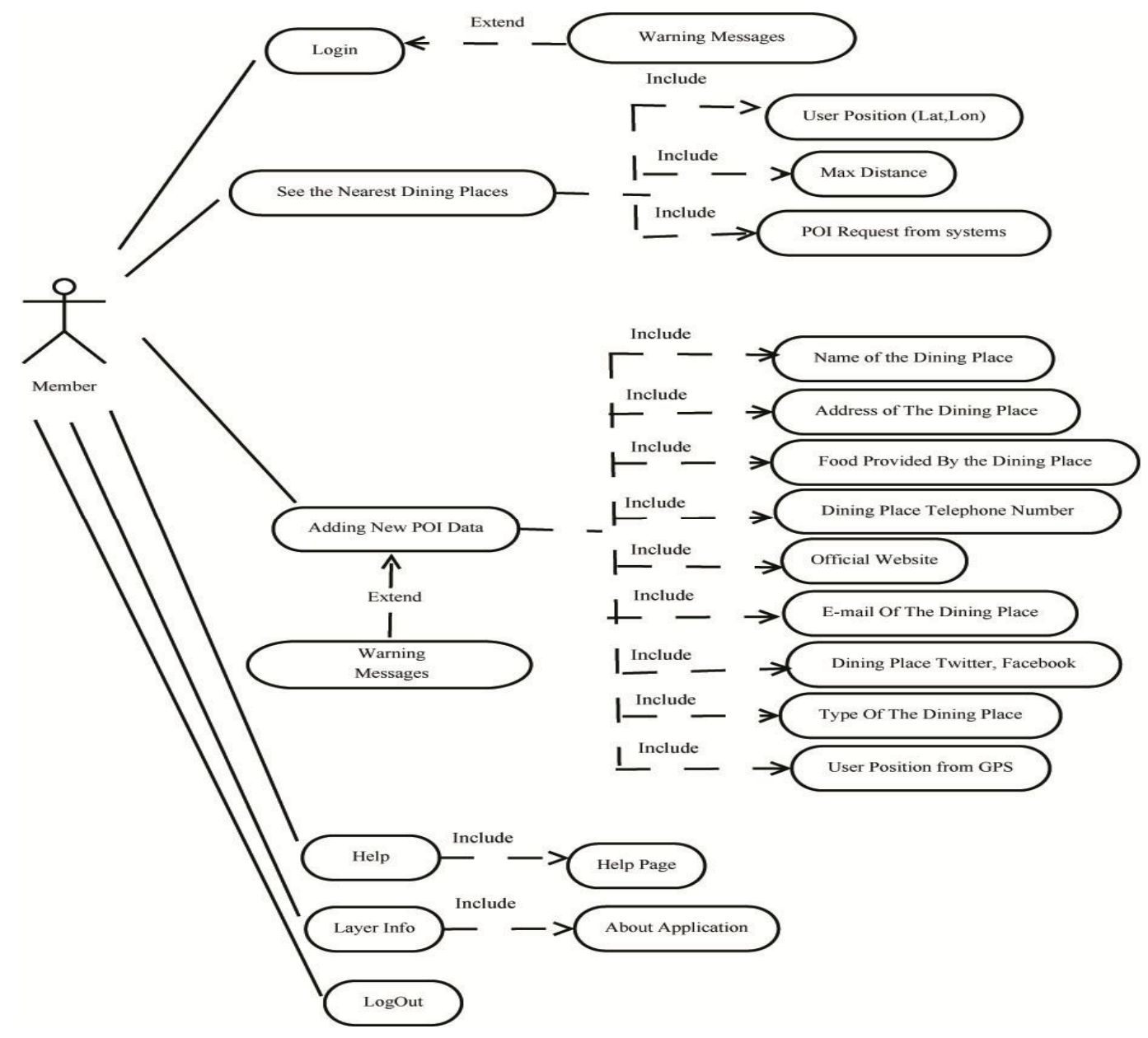

Figure 2 : Usecase diagram for Member 


\subsection{Usecase Diagram for Non Member}

As previously described user who acts as non member / not listed user, then the inaccessibility of facilities different from a user belonging a member. From the Figure 3 it can be seen that non member can do :

- Seeing the nearest dining places where its parameters are the coordinates of the user position, the maximum distance specified by user and POI Request from the system.

- Sign Up as a member with the required parameters are username and password.

- Help to open the Help Page.

- Layer Info to open pages that describes the application.

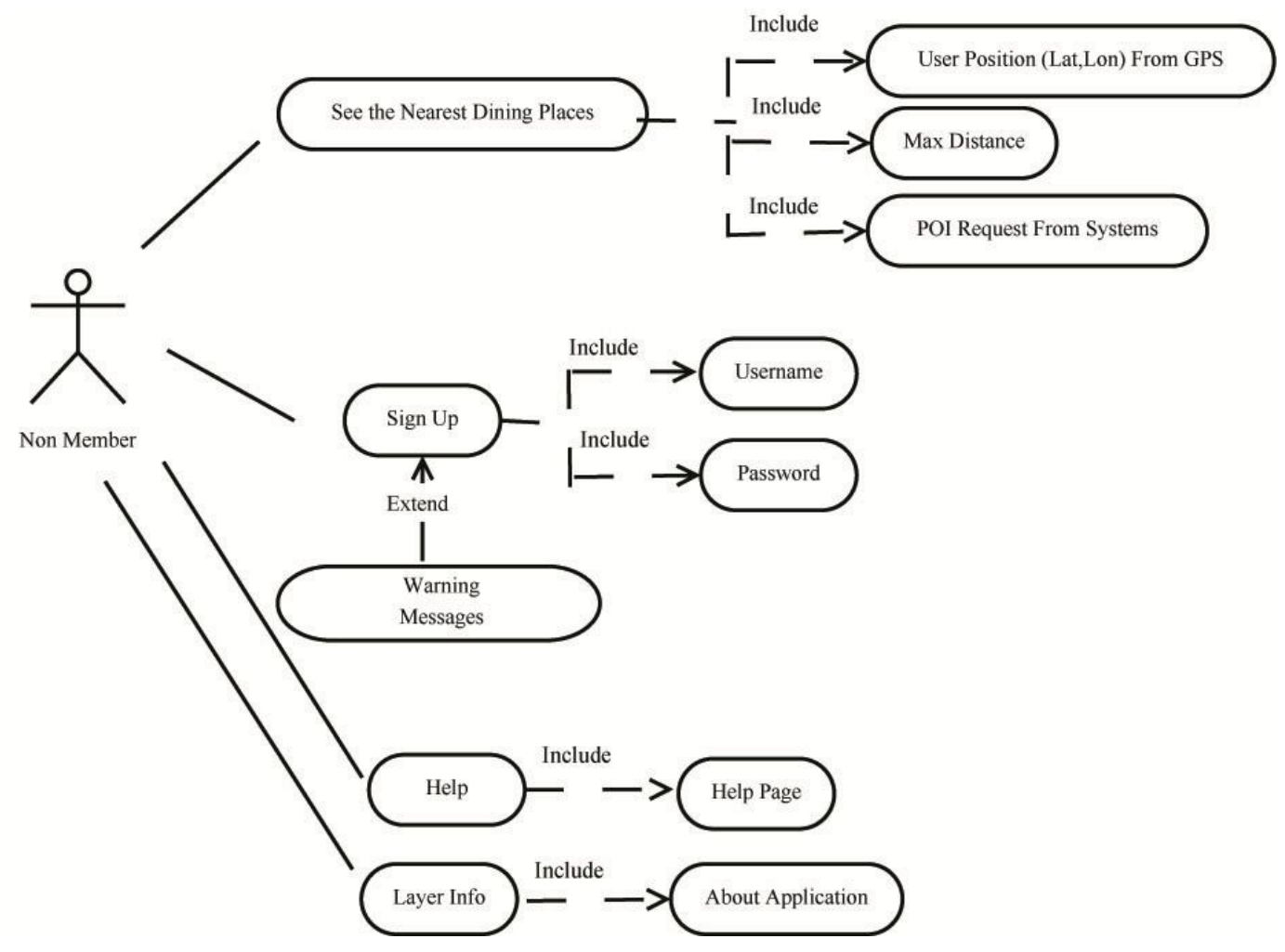

Figure 3 : Usecase Diagram for Non Member

\subsubsection{Class Diagram}

Class diagrams are used to describe relationships between classes in a system that is being made and how they collaborate in order to achieve a goal.

\subsection{Class Diagram for Admin}

It can be seen from the Figure 4 that the Admin Class Diagram has 3 classes namely Login, Data_POI and Action. The multiplicity between Admin and Login class is one-to-one, while the multiplicity between Login with Data POI and Action class is one-to-many. It is because, one Admin can see, insert, delete and edit many of Data POI and Action. 
Advanced Computing: An International Journal (ACIJ ), Vol.4, No.4, July 2013

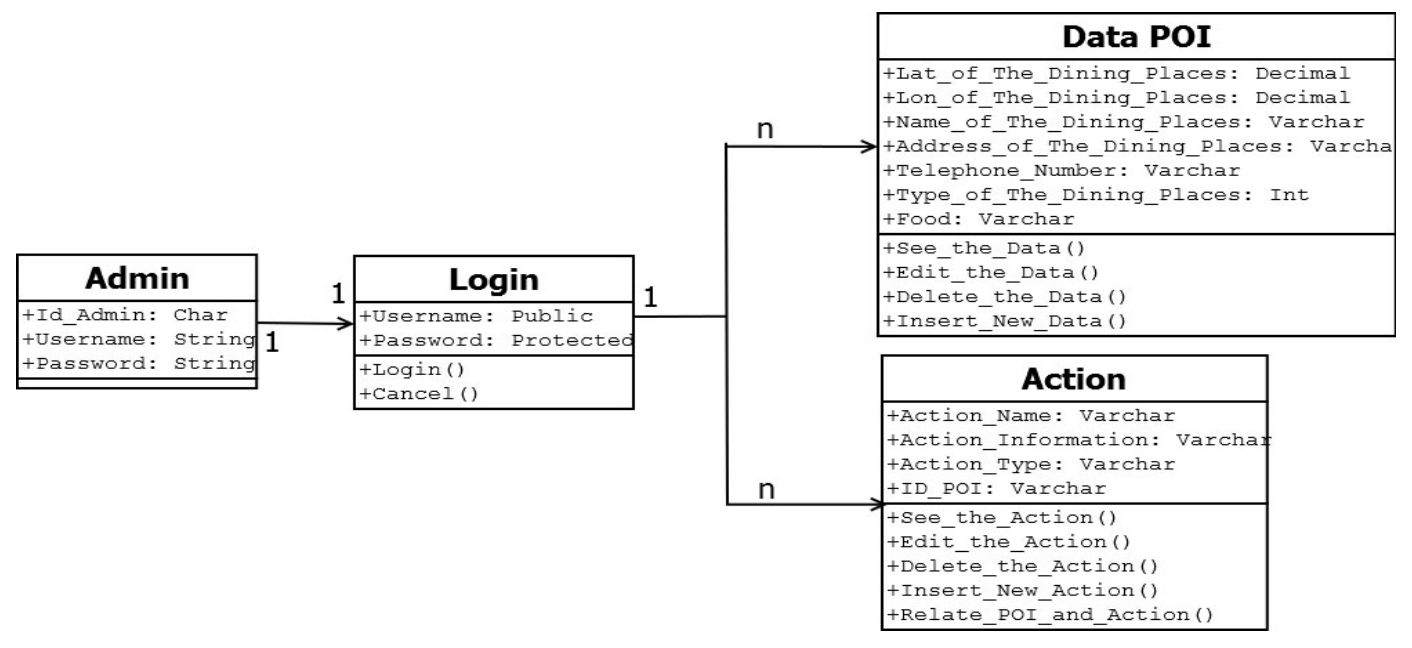

Figure 4 : Class Diagram for Admin

\subsection{Class Diagram for Member}

In this section there are 6 classes diagram because member has many facilities on the application. The multiplicity between Member and Login class is many-to-one because there are many users / members who can login, while the the multiplicity between Login and Dining Places, New POI, Help, Layer Info and LogOut is many-to-many. This can be seen clearly in the Figure 5 below.

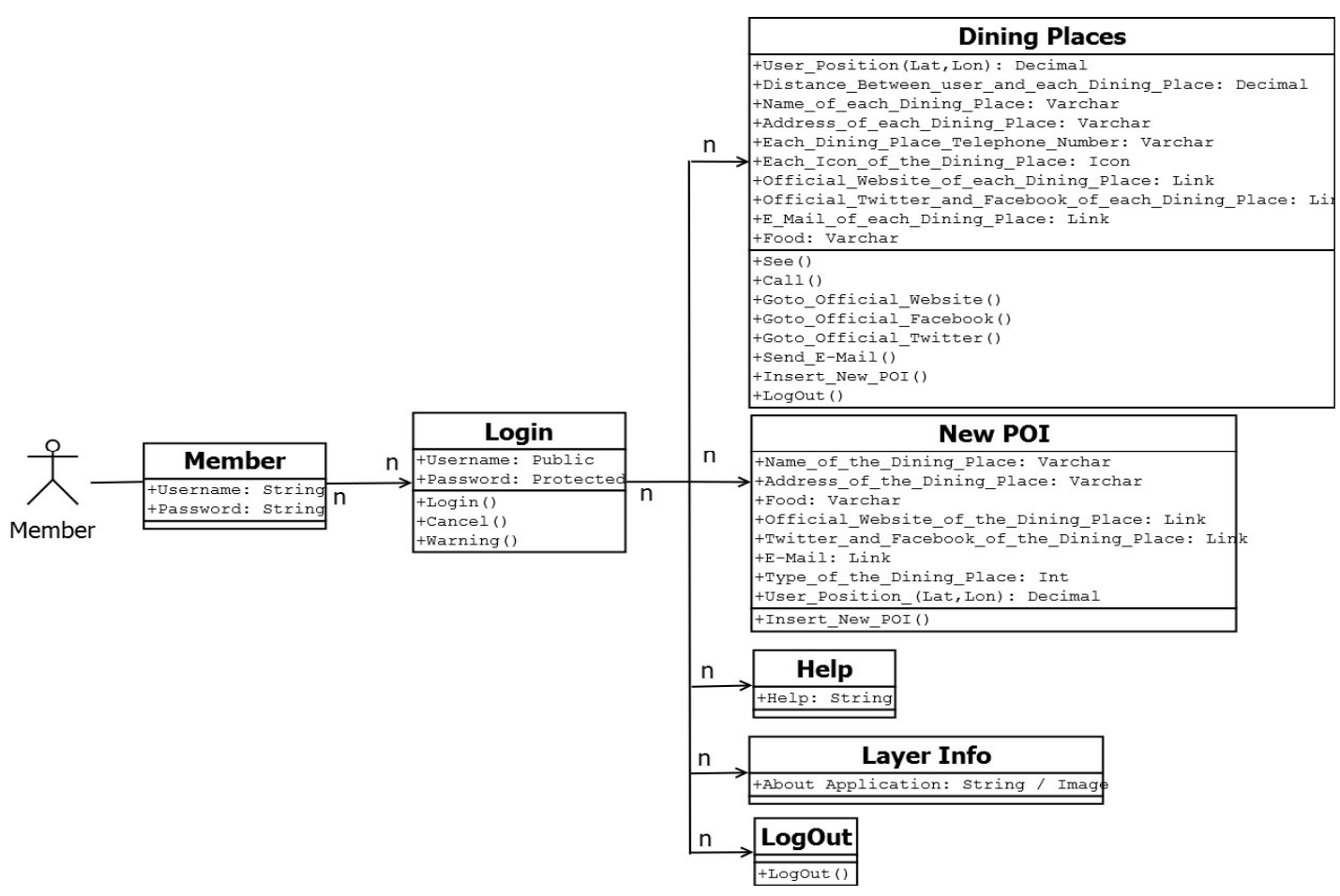

Figure 5 : Class Diagram for Member 


\subsection{Class Diagram for Non Member}

At Non Member Class Diagram there are 10 class. Where 3 pairs class are the same class. This is because there are the same class between a Non Member with the Member (which comes from a Non Member who register to become a Member). 3 pairs classes are Dining Places Class, Help Class and Layer Info Class. While 4 other class are Sign Up Class, Login Class, New POI Class and LogOut Class. From the Figure 6 can be known that :

- The multiplicity between Not Member and Dining Places Class, Sign Up Class, Help Class and Layer Info Class is many-to-many.

- The multiplicity between Sign Up Class with Login Class is many-to-many.

- While the multiplicity between Login Class with Dining Places Class, New POI Class, Help Class, Layer Info Class and LogOut Class is many-to-many. Because many users can login and be able to do a lot of menus choices in the application.

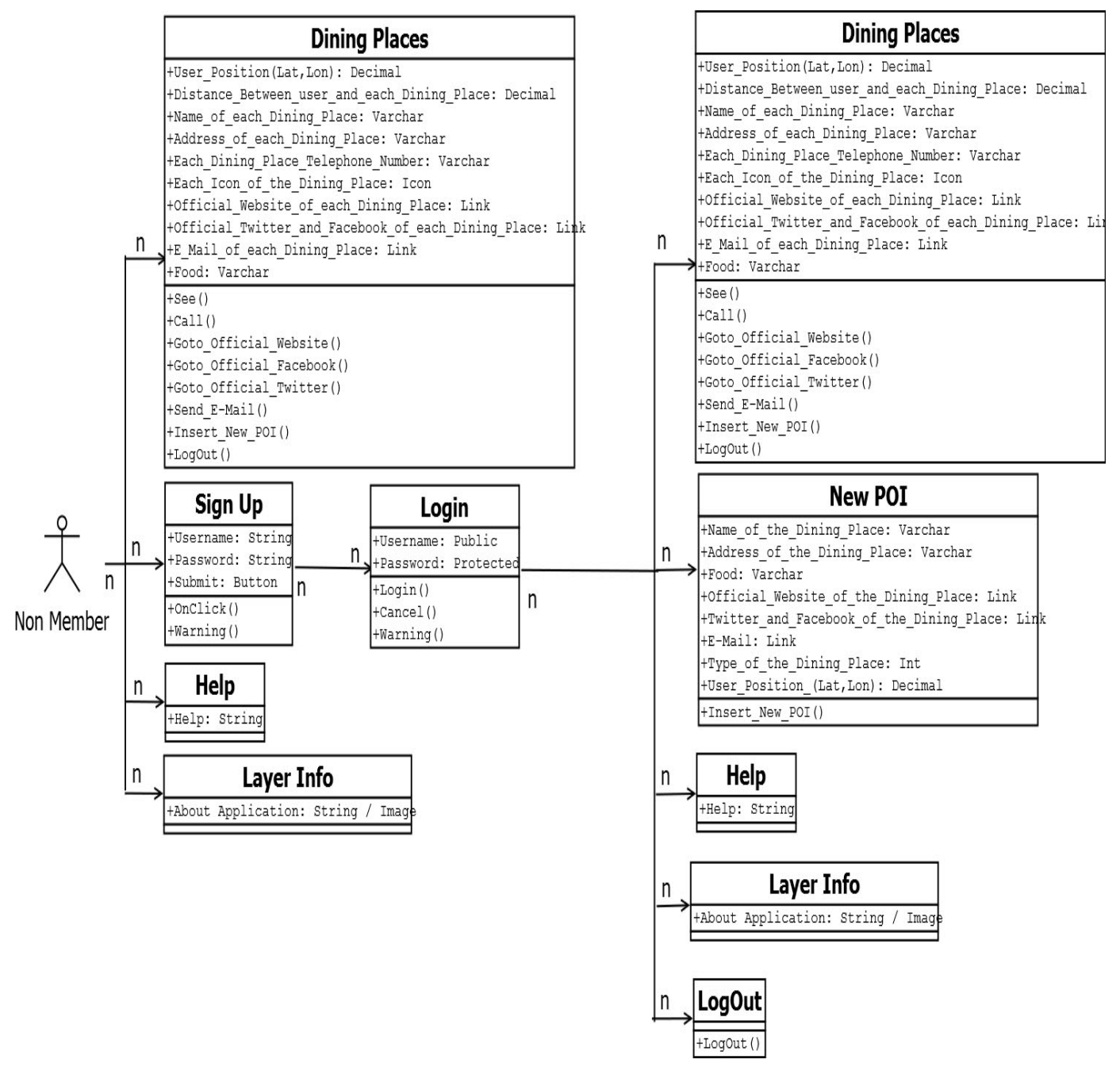

Figure 6 : Class Diagram for Non Member 
Advanced Computing: An International Journal (ACIJ ), Vol.4, No.4, July 2013

\subsubsection{Flowcharts}

\subsection{Member's Flowchart}

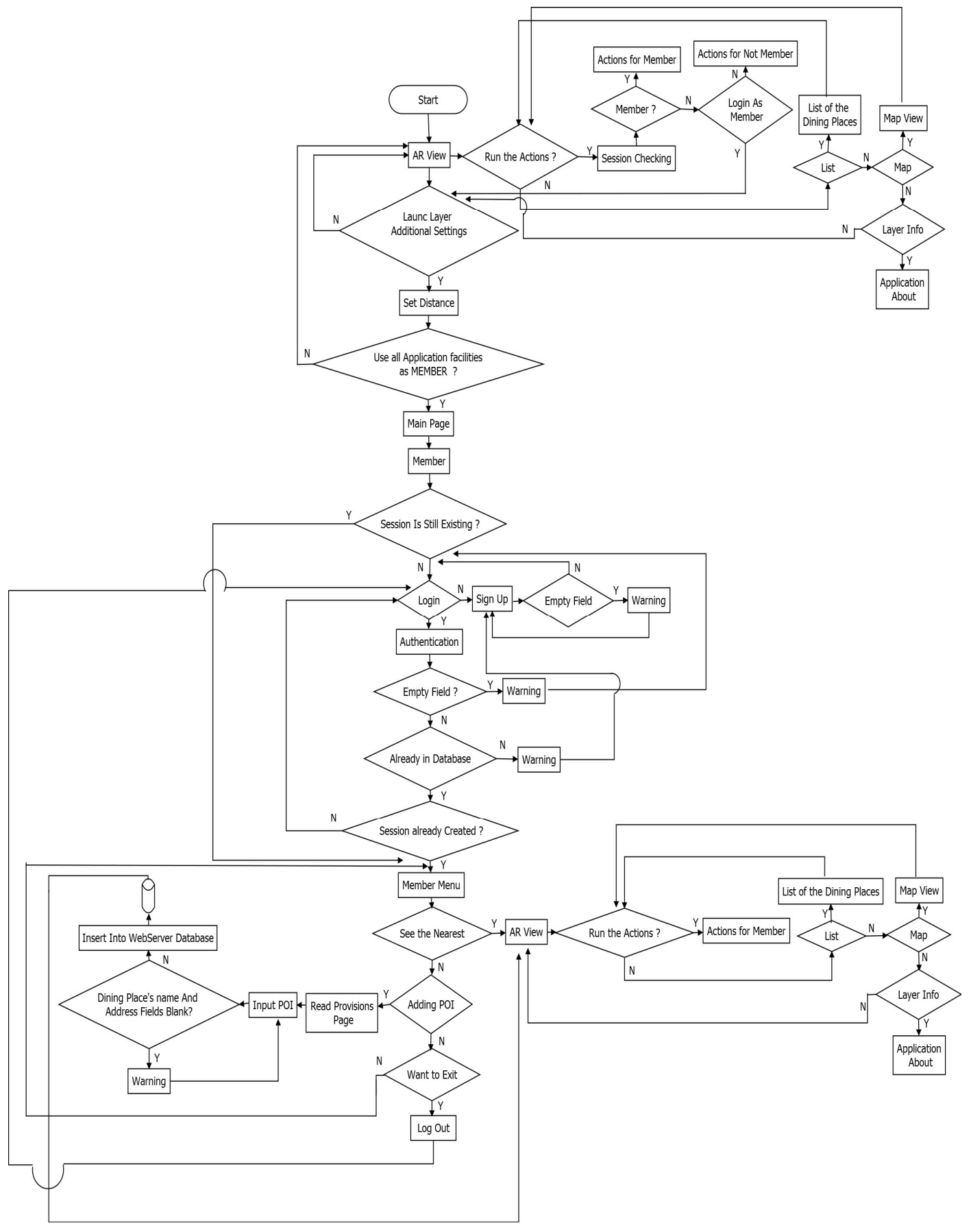

Figure 7 : Flowchart of Application's Member 
Advanced Computing: An International Journal (ACIJ ), Vol.4, No.4, July 2013

\subsection{Non Member's Flowchart}

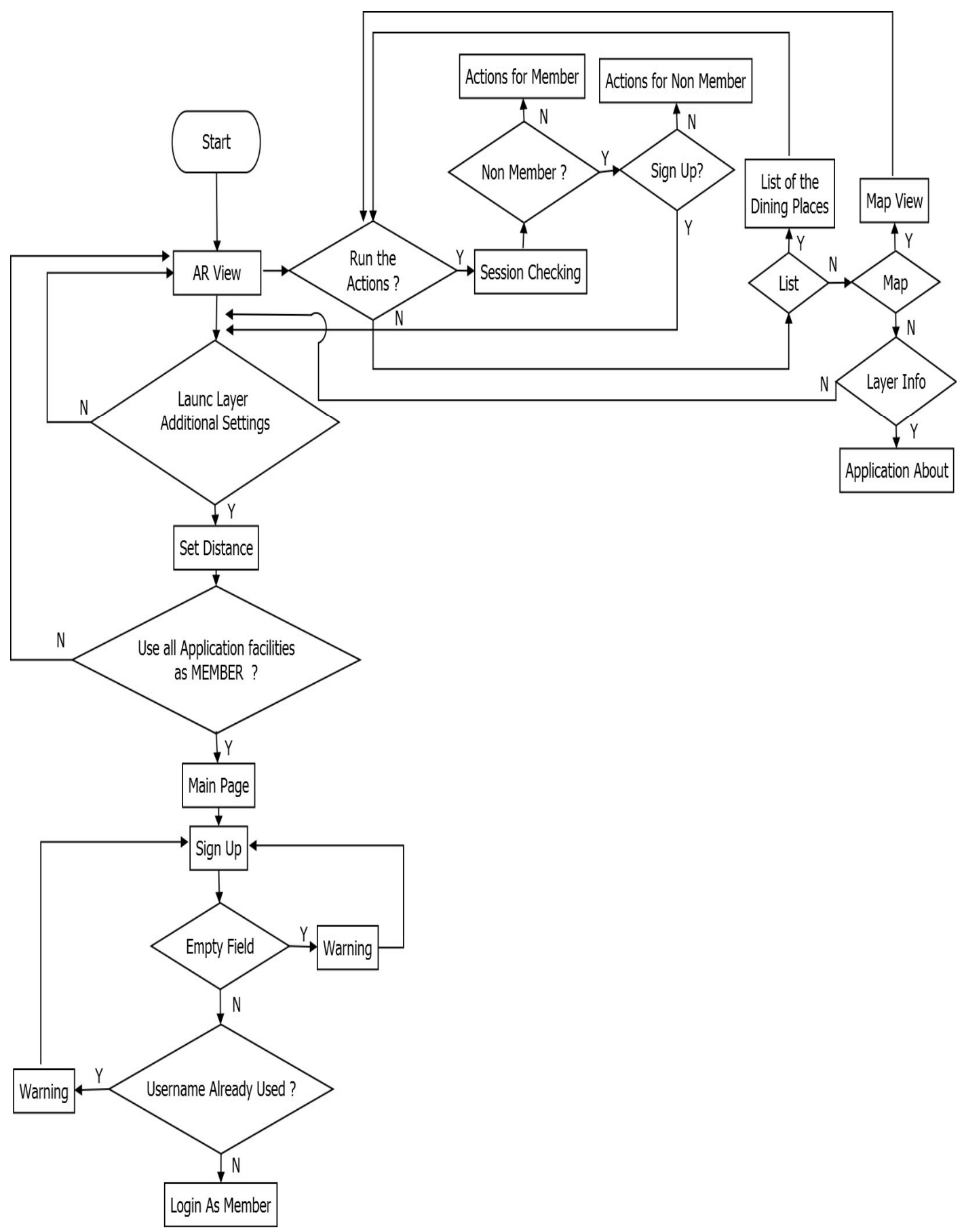

Figure 8 : Flowchart of Application's Non Member 


\subsection{Sign Up Flowchart}

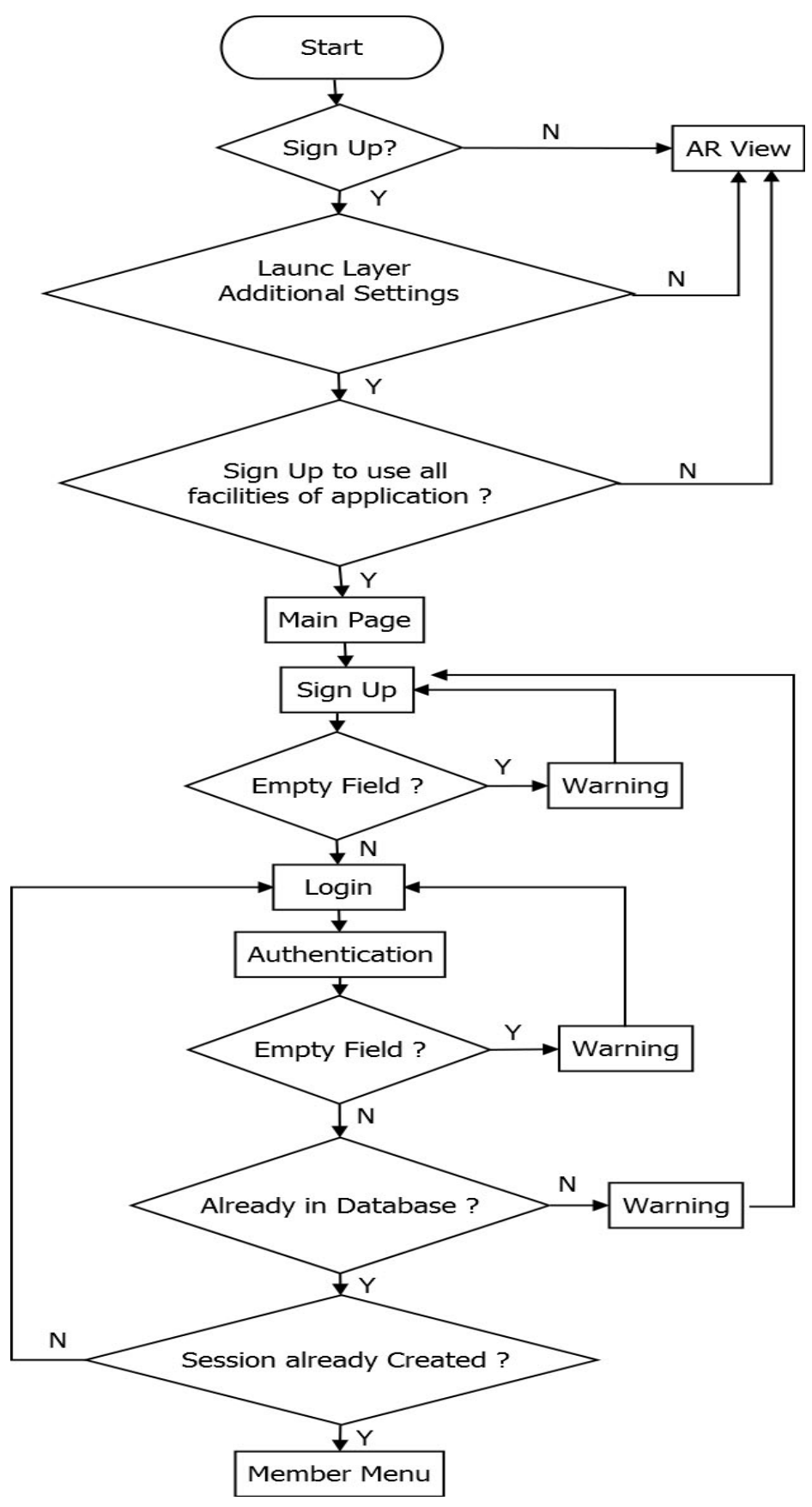

Figure 9 : Application's Sign Up Flowchart

\subsubsection{Layout Design}

Layout design consists of several pages was made to facilitate the user in using the application. This section explains how the design of each page such as Main Page, Login Page, Sign Up Page, Member Menu Page, Provisions Page, New POI Page and Help Page. 


\subsubsection{Main Page}

This is the Main Page where the user can use all the facilities provided by the application (facilities for a Member). In this Page there are 2 command buttons. If the user pressing Button1 then if the session is still user will be taken to the Member Menu Page whereas if the session does not exist it will be to the Login Page. By pressing Button2 as the Sign Up button user will be taken to the Sign Up Page. Figure 10 is the design of Main Page.

\section{Background}

TITLE

\section{Button 1}

\section{Button 2}

Figure 10 : The design of Main Page

\subsubsection{Login Page}

The Login Page is the page where user must input username and password correctly to enter into the Member Menu Page in this application by pressing Button1 as the Login button. This form shows the background image, and some texts. User can fill in Input Text1 with Username and in Input Text 2 with Password. When user clicks the button user input will be process to know if any errors happened and if any errors occurs by user then Text 3 will show a Warning Messege. In this Login Page, there is a hyperlink text (Hyperlink Text1) where if the user clicks it will immediately be heading to Sign Up Page. Figure 11 is the design of Login Page.

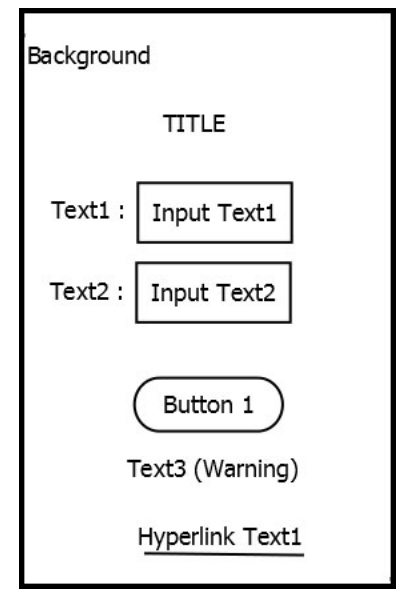

Figure 11 : The design of Login Page 


\subsubsection{Sign Up Page}

The Sign Up Page is the form where user must input username and password correctly to be a member of this application by pressing Button1 as the Submit button. When user clicks the button user input will be process to know if any errors happened and insert the data into database. If any errors occurs by user then Text 3 will show aWarning Messege. This form shows the background image and some texts. User can fill in Input Text1 with Username and in Input Text2 with Password. Figure 12 is the design of Sign Up Page.

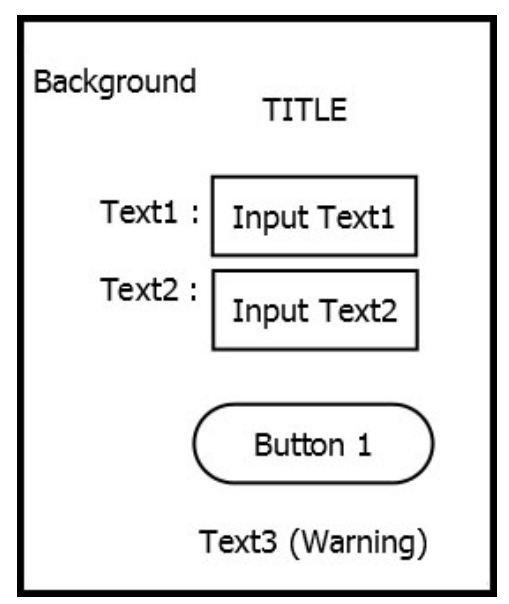

Figure 12 : The design of Sign Up Page

\subsubsection{Member Menu Page}

After the MEMBER button pressed by the user in Main Page (If session is exist) / after user success login in Login Page, the next stage is the Member Menu Page. This Page contains of title, background image, some texts and buttons. Button1 is a button if user want to LogOut. Button2 is a button if user want to see the nearest dining places / POI. If user clicked it, AR View will appear. Button3 is a button if user / member want to add new POI (dining place) into application database. If user clicked it, Provisions Page will appear. Figure 13 is the design of Member Menu Page.

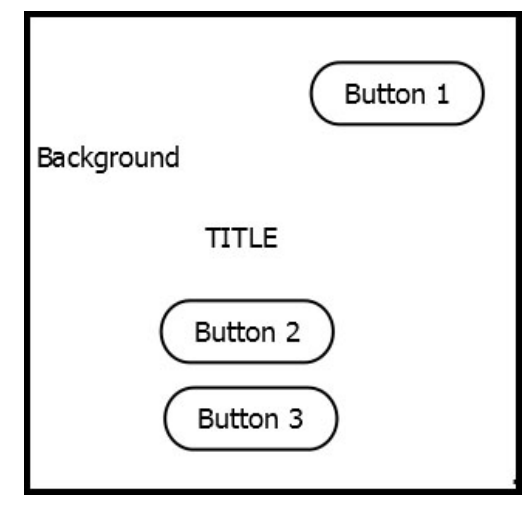

Figure 13 : The design of Member Menu Page 


\subsubsection{Provisions Page}

The page contains the points that must be considered when user want to add new POI data to application database. This page shows the background image and some texts. Title1 as the Title of the Page. Button1 is a button user have to clicks after read the provisions and will heading to New POI Page. Figure 14 is the design of Provions Page.

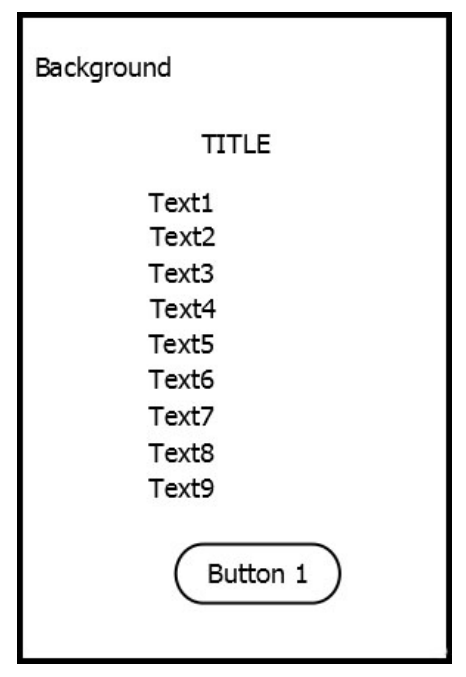

Figure 14 : The design of Provisions Page

\subsubsection{New POI Page}

This is the page that made the difference of facility that can be used by member besides logout. This form shows the background image and some texts. Tittle is the form's name. In this form user can input POI data that needed for the fulfillment of important information of the POI. User can fill in Input Text1 with Name of dining place, Input Text2 with Address of the dining place, Input Text 3 with Food Provided by the dining place, Input Text 4 with Phone Number of the dining place, Input Text5 with Official Website of the dining place, Input Text6 with E-Mail address of the dining place, Input Text7 with Official Facebook of the dining place and Input Text8 with Official Twitter of the dining place. Select List to input Type of the dining place. When user clicks the Button1 as the Submit button, user input will be process to know if any errors happened and insert the data into database. If any errors occurs by user then Text10 will show a Warning Messege. Figure 15 is the design of New POI Page. 
Advanced Computing: An International Journal (ACIJ ), Vol.4, No.4, July 2013

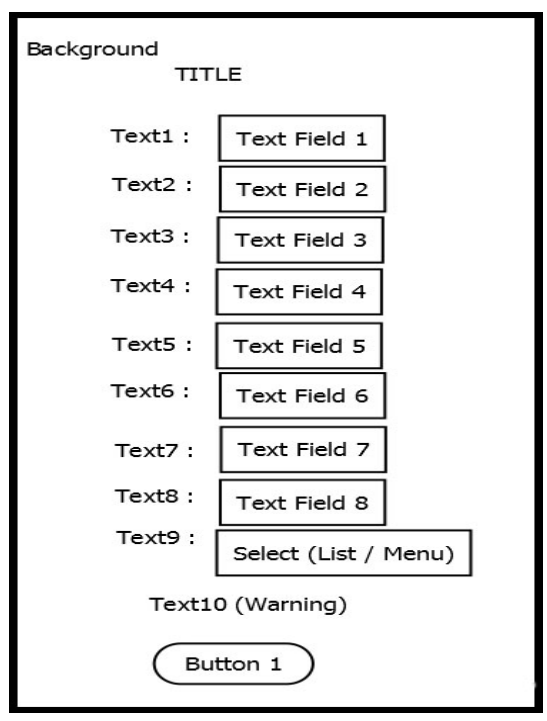

Figure 15 : The design of New POI Page

\subsubsection{Help Page}

The page contains the points that must be considered in using this application. This page shows the background image and some texts. Title1 as the Title of the Page. Button1 is a button user have to clicks after read the points and will return to the AR View. Figure 16 is the design of Help Page.

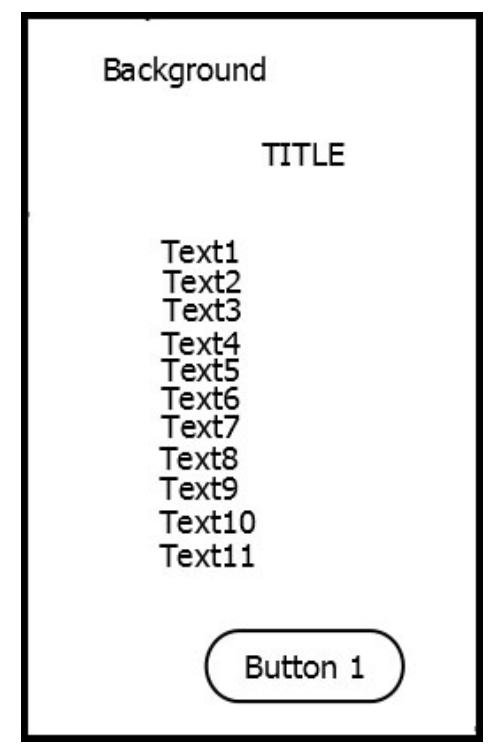

Figure 16 : The design of Help Page

\section{Conclusions}

The development of this application aims to help users find the preferred dining place which being around them along with supporting information. Besides just display information, the application is designed to be a Reciprocal application which can do something based on the input received from the user either in the New POI Page / information but also input method such as click. 


\section{REFERENCES}

[1] Wikipedia, "Location-based service". [Online]. Available: http://en.wikipedia.org/wiki/Locationbased_service

[2] B. Jiang and X. Yao, "Location-based services and gis in perspective," Computers, Environment and Urban Systems, vol. 30, pp. 712-725, 2006.

[3] R. T. Azuma, "A survey of augmented reality," Teleoperators and Virtual Environments, vol. 6, pp. 355-385, 1997.

[4] L. Madden, Wrox,Professional Augmented Reality Browsers for Smartphones [programming for junaio,layar and Wikitude]. Wiley Publishing, 2011.

[5] Layar, "User Support". [Online]. Available: http://www.layar.com/user-support/

[6] Wikipedia, "Class diagram". [Online]. Available: http://en.wikipedia.org/wiki/Class_diagram

[7] Wikipedia, "Flowchart". [Online]. Available: http://en.wikipedia.org/wiki/Flowchart

\section{Authors}

\section{Farhat, ST.}

Has graduated as a Bachelor of Engineering from Department of Informatics Engineering at the Faculty of Industrial Technology, Gunadarma University in 2013.Now, pursue graduate studies of master degree in Department of Information Systems Management, Gunadarma University. Since 2011, became assistant of the Computerized Development Institute (LePKom) for Database Laboratory at the Gunadarma University.

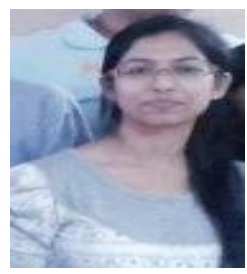

